\title{
Ophthalmic Manifestations and its Correlation to Platelet Count in Dengue Patients in a Tertiary Care Hospital of Eastern India
}

\author{
Dibya Prabha ${ }^{1 *}$, Yuvraj Lahre ${ }^{2}$, Lily Kumari ${ }^{3}$, Pawan Kumar $^{4}$, Apeksha Singh $^{5}$ \\ ${ }^{1}$ Senior Resident, Department of Ophthalmology, PMCH, Dhanbad, Jharkhand, India \\ ${ }^{2}$ Senior Resident, Department of Medicine, MGM Medical College and Hospital, Jamshedpur, Jharkhand, India \\ ${ }^{3}$ Senior Resident, Department of Medicine, PMCH, Dhanbad, Jharkhand, India \\ ${ }^{4}$ Senior Resident, Department of Medicine, Dumka Medical College and Hospital, Dumka, Jharkhand, India \\ ${ }^{5}$ Senior Resident, Department of Dermatology, PMCH, Dhanbad, Jharkhand, India
}

Article History

Received: 11.12 .2020

Accepted: 21.12 .2020

Published: 24.12.2020

Journal homepage: https://www.easpublisher.com

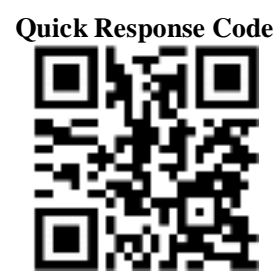

Abstract: Purpose: To determine clinical spectrum of ophthalmic manifestations and its correlation to platelet count in dengue patients. Materials and methods: Consecutive patients of dengue disease who presented during June 2019 to August 2019 were evaluated. Complete ocular examinations were done. Results: Out of 92 patients, $61.95 \%$ were male, $38.04 \%$ were female. Mean age of presentation was 32.65 years. Number of patients with ocular symptoms was $33.69 \%$ \& with ocular signs was $45.65 \%$. Subconjunctival haemorrhage was the commonest ocular findings (25\%). Posterior segment findings were present in $11.95 \%, 7.60 \%$ had retinal haemorrhage. $40.50 \%$ DF, $72.72 \%$ DHF patients and $100 \%$ DSS had ocular findings. Ocular findings were present in $19.60 \%$ in patients with platelet count $>1$ lakh \& $83.33 \%$ in patients with platelets count $<20000$. Conclusion: Ophthalmic manifestations are not uncommon but mostly under observed, so ocular examinations in all dengue cases is advised to prevent visual complications.

Keywords: Ophthalmic manifestations, Dengue, Subconjuctival haemorrhage, platelets.

Copyright (C) 2020 The Author(s): This is an open-access article distributed under the terms of the Creative Commons Attribution 4.0 International License (CC BY-NC 4.0) which permits unrestricted use, distribution, and reproduction in any medium for non-commercial use provided the original author and source are credited.

\section{INTRODUCTION}

Dengue is vector borne viral disease. Causative virus belongs to Family Flaviviridae which includes four different serotypes (DEN1, DEN2, DEN3, DEN4)[1]. Dengue is one of the major public health problems in tropic and subtropic nations. Due to increasing population, global warming, increased urbanization, inefficient mosquito control, inadequate health care facilities etc there is rise in dengue infection worldwide [2]. First reported case of dengue in India was in Madras and first epidemic of dengue in India was occurred in Calcutta [3].

Dengue is transmitted by bite of female mosquito Aedes aegypti and Aedes albopictus [4]. Transmission is mostly in rainy season. Etiopathological factors for dengue infections are viral replication in macrophages, immunological reaction against viral antigens [5]. Clinically it is characterised by acute onset of high grade fever which last for 3 days to weeks, malaise, cough, vomiting, headache, bodyache, retro-orbital pain, joint pain, skin rash etc[6]. Other clinical manifestations are due to complications of thrombocytopenia.
Dengue is clinical diagnosis in most of cases, but can be confirmed by with laboratory test such as polymerase chain reaction PCR, immunoglobulin M (igM, IgG) [7]. Dengue infection is usually self limiting. Recovery from one serotype provides lifelong immunity from that serotype only but not from other serotype so sequential infection from other serotype may cause serious systemic disease such as dengue hemorrhagic fever, dengue shock syndrome.

With increase in number of dengue cases, ocular manifestations associated with dengue also increased. It varies from mild conjunctival congestion to vision threatening complications [8]. So our aim is to highlight ocular manifestations of dengue and to find out correlation with platelet count.

\section{Materials And Methods}

- A cross- sectional analytical study of 92 patients with dengue disease who were admitted in dengue ward of department of medicine during June 2019 to August 2019 was done after informed consent and ethics clearance. Inclusion criteria for study 
were patients who were serologically positive for NS1and we excluded patients who were having concurrent febrile illness from malaria etc, DM, HTN, patients in which informed consent could not be obtained. Detailed clinical history, systemic examination, Laboratory reports of $\mathrm{CBC}$, platelet count, serological assay of NS1, IgM, Ig G were noted. Malaria ruled out. Ocular examinations such as BCVA using Snellen chart, Pupillary light response, anterior segment examination using slitlamp, fundoscopy with indirect ophthalmoscopy were done. Fundus photos were documented in patients with retinal findings. OCT done in patients with maculopathies. A follow up telephone call was made 2 weeks after the onset of fever to rule out any onset of new visual symptoms.

- A correlation study between platelet count and ocular manifestations was done using Pearson correlation coefficient. All statistical analysis were done using SPSS by IBM, version 19.0

\section{RESULTS}

A total of 92 patients of age 15-60 years diagnosed as dengue were enrolled in our study out of which $57(61.95 \%)$ were male and $35(38.04 \%)$ were female[Table1]. Mean age of presentations was 32.65years (15-60). Minimum interval between onset of fever and eye examination was 4 days. Patients mainly belong to lower socioeconomic status. In our study all cases presented with fever, myalgia $(76.3 \%)$, headache $58.34 \%$. other complains were nausea, pain abdomen, loose motions etc. Platelet counts in our study varied widely, lowest being 9000 and median count were 80000 .

Ocular findings were seen in $42(45.65 \%)$ [Table1]. Out of which anterior segment findings were seen in $33.69 \%$ and posterior segment findings were in $11.95 \%$. Among anterior segment findings subcounjunctival haemorrhages were seen in $25 \%$ which was the most commonly seen ocular findings, chemosis in $7.60 \%$, lid edema $1.08 \%$. Among posterior segment findings retinal haemorrhages were most commonly seen in $7.06 \%$, exudates $1.08 \%$, disc edema $3.26 \%$ [Table1].

Table-1: Ocular findings in patients of dengue

\begin{tabular}{|l|l|l|}
\hline Ocular findings & Number & Percentage \\
\hline Anterior segment findings & 31 & $33.69 \%$ \\
\hline Subconjunctival hemorrhage & 23 & $25 \%$ \\
\hline chemosis & 7 & $7.60 \%$ \\
\hline Lid edema & 1 & $1.08 \%$ \\
\hline Posterior segment findings & 11 & $11.95 \%$ \\
\hline Retinal hemorrhage & 7 & $7.60 \%$ \\
\hline exudates & 1 & $1.08 \%$ \\
\hline Disc edema & 3 & $3.26 \%$ \\
\hline
\end{tabular}

In cases of dengue fever $40.50 \%$ patients had ocular findings, in DHF, $72.72 \%$ and in DSS all $100 \%$ were having ocular findings [Table2][ Figure 1].

Table-2: Ocular manifestations in different dengue patients

\begin{tabular}{|l|l|l|l|l|}
\hline & DF & DHF & DSS & Total \\
\hline With ocular findings & $32(40.50 \%)$ & $8(72.72 \%)$ & $1(100 \%)$ & $42(45.65 \%)$ \\
\hline Without ocular findings & $47(59.49 \%)$ & $3(27.27 \%)$ & 0 & $53(54.34 \%)$ \\
\hline Total patients & 79 & 11 & 1 & 92 \\
\hline
\end{tabular}

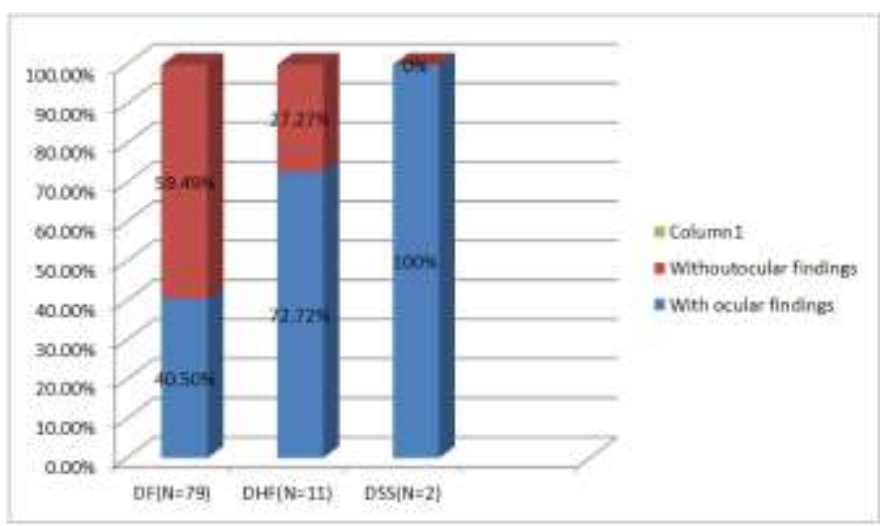

Fig-1: showing percentage of patients with ocular findings in different dengue patients 
Ocular findings were present in $10(19.60 \%)$, $13(61.90 \%), 14(82.35 \%), 5(83.33 \%)$ in patients with following Platelets count $>100000,100000-50000,50 \mathrm{k}-$ $20 \mathrm{k}$ and $<20000$ [Figure2].

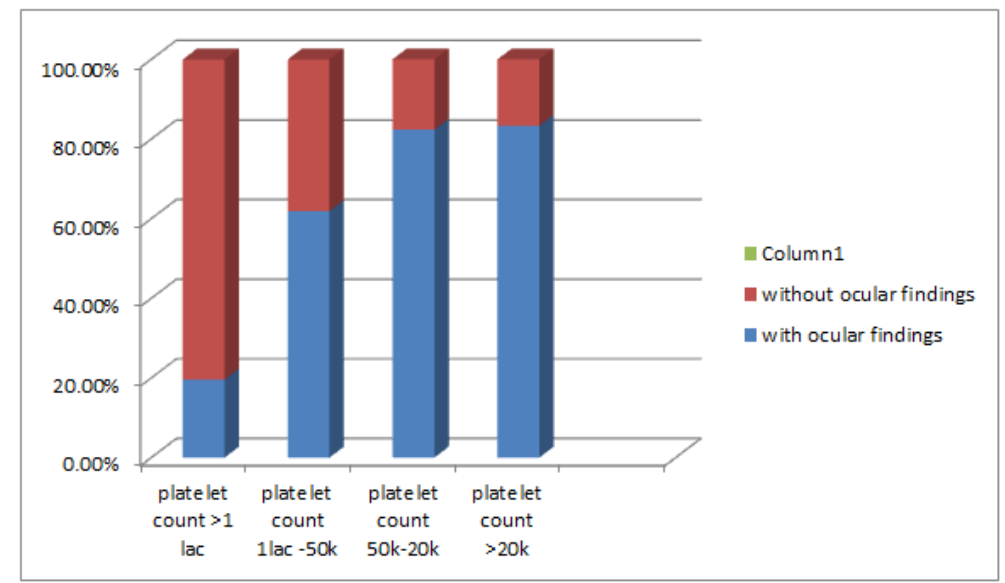

Fig-2: Platelet count and correlation with ocular manifestations

\section{Discussion}

Presently uprise surge in dengue cases are mainly attributable to increased population, increased global temperature, inefficient mosquito control and inadequate health facilities [2, 4, 7]. In our study we found mean age of presentation 32.65years with male preponderance which is similar to other studies $[9,10]$. There are very few studies that correlated blood parameters with ocular findings. We reported $45.65 \%$ patients had ocular manifestations also noted in study by [11]. Among anterior segment findings $33.69 \%$, SCH was most common in $25 \%$ cases which are almost similar to study done by [12]. Only $11.95 \%$ patients had posterior segment involvement which is also similar to Kapoor et al. [11]. Retinal haemorrhages, exudates, disc edema were most common posterior segment findings. In our study we found $40.50 \%$ patients of dengue fever had ocular findings while $72.72 \%$ and $100 \%$ patients had ocular findings in DHF and DSS which was seen in study done by [13]. In our study we found ocular findings increases with decrease in platelet count. This is also noted by study done by [13] while [14] reported no significant association between thrombocytopenia and hemorrhagic manifestations. In our study, a statistically significant correlation was seen between platelet count and ocular manifestations; this was supported by previous study done by [15].

Our limitation of study was we haven't follow up patients so cannot describe time taken to resolve as our study was non interventional. Smaller sample size, single hospital based study, only hospitalised patients were considered are other limitations which need to improve.

\section{CONCLUSION}

With increase in cases of dengue infection, ophthalmic manifestations expected to rise. Patients with marked thrombocytopenia are more predisposed to ocular complications. It may be simple $\mathrm{SCH}$ or retinal haemorrhage which can impair vision. Physician should refer patients of DHF, DSS, with deteriorated blood parameters to ophthalmologist for early detection of ocular manifestations.

\section{REFERENCES}

1. Kurane, I. (2007). Dengue hemorrhagic fever with special emphasis on immunopathogenesis. Comparative immunology, microbiology and infectious diseases, 30(5-6), 329340.

2. World Health Organization, Special Programme for Research, Training in Tropical Diseases, World Health Organization. Department of Control of Neglected Tropical Diseases, World Health Organization. Epidemic, \& Pandemic Alert. (2009). Dengue: guidelines for diagnosis, treatment, prevention and control. World Health Organization.

3. Gupta, N., Srivastava, S., Jain, A., \& Chaturvedi, U. (2012). Dengue in India. The Indian journal of medical research, 136(3), 373-390.

4. Gubler, D. J. Dengue and dengue hemorrhagic fever. Clin Microbial Rev.[Internet]. 1998 [citado 13 de 2015]; 11 (3): 480-96.

5. Whitehorn, J., \& Simmons, C. P. (2011). The pathogenesis of dengue. Vaccine, 29(42), 72217228.

6. Dhar, S. (2017). A Study on the Clinicopathological Profile and Outcome of a Dengue Epidemic in Western Odisha. International Journal of Medical Science and Innovative Research, 2(4):13- 20. 
7. Guzman, M. G., Halstead, S. B., Artsob, H., Buchy, P., Farrar, J., Gubler, D. J., \& Nathan, M. B. (2010). Dengue: a continuing global threat. Nature reviews microbiology, 8(12), S7S16.

8. Yip, V. C. H., Sanjay, S., \& Koh, Y. T. (2012). Ophthalmic complications of dengue fever: a systematic review. Ophthalmology and therapy, 1(1), 2.

9. Haritoglou, C., Scholz, F., Bialasiewicz, A., \& Klauss, V. (2000). Okuläre Manifestation bei Dengue-Fieber. Der Ophthalmologe,97(6), 433436.

10. Intizar Hussain, F. C. P. S., Afzal, F., \& DOMS, A. S. (2012). Ophthalmic manifestation of dengue fever. AN OFFICIAL JOURNAL OF PESHAWAR MEDICAL COLLEGE, 10(1), 93.

11. Kapoor, H. K., Bhai, S., John, M., \& Xavier, J. (2006). Ocular manifestations of dengue fever in an
East Indian epidemic. Canadian journal of ophthalmology, 41(6), 741-746.

12. Rani Sujatha, S. N., Nazlin, A., \& Prakash, S. (2015). Ocular manifestations of dengue fever. Int J Med Sci Public Health, 4(5), 690-3.

13. Dhar, S. K., Samant, S., Mishra, S., Tripathy, D., Shah, R. D., \& Prasad, R. C. Spectrum of Ophthalmic Manifestations of Dengue: Our Experience in a Tertiary Care Centre.

14. Gomber, S., Ramachandran, V.G., Kumar, S. (2001). Haematological observations and diagnostic markers in dengue haemorrhagic fever-a reappraisal. Indian Pediatr, 38:477-81.

15. Nagaraj, K. B., Jayadev, C., Yajmaan, S., \& Prakash, S. (2014). An unusual ocular emergency in severe dengue. Middle East African Journal of Ophthalmology, 21(4), 347. 